\title{
The LeMMINGs e-MERLIN survey of nearby galaxies
}

\section{Rob Beswick (co-PI)*, Megan Argo, Ruth Evans}

JBCA, The University of Manchester

E-mail: robert.beswick@manchester.ac.uk

\section{Ian McHardy (co-PI), David Williams}

University of Southampton

\section{Jonathan Westcott}

University of Hertfordshire

\section{on behalf of the LeMMINGs collaboration}

LeMMINGs is a large e-MERLIN Legacy ${ }^{\dagger}$ survey, awarded 810 hours of observing time over the first five semesters of e-MERLIN operations. The observational aim of the project is to image a complete sample of nearby galaxies, encompassing all galaxy types, at sub-arcsecond (few pc) angular resolution and $\mu$ Jansky sensitivity. The main science themes for the survey are to measure star-formation activity in the sample galaxies, make a complete census of accretion activity in galaxies of all types, and to carry out a parsec-scale suvey of the cold ISM via absorption and emission lines. The programme includes multi- $\lambda$ follow-up, and will ultimately provide a public legacy data set of use to the wider astronomical community.

12th European VLBI Network Symposium and Users Meeting,

7-10 October 2014

Cagliari, Italy

\footnotetext{
*Speaker.

${ }^{\dagger}$ http://www.e-merlin.ac.uk/legacy
} 


\section{The science}

LeMMINGs, the Legacy e-MERLIN Multi-band Imaging of Nearby Galaxies survey, is built around three core science themes: 1 . measuring star-formation activity, 2 . making a complete census of AGN activity and jet structures in galaxies of all types, measure radio LF for different galaxy types, properly define $\mathrm{L}_{\mathrm{R}} / \mathrm{L}_{\mathrm{X}} / \mathrm{M}$ 'fundamental plane', and 3. a serendipitous parsec-scale imaging survey of the cold ISM via atomic and molecular absorption/maser emission.

Star-formation: the LeMMINGs observations will detect and resolve RSNe/SNRs at moderate distance for all of the galaxies in the sample. In addition, targeted deeper observations on a sub-sample will allow us to detect the weaker radio emission from planetary nebulae, HII regions and super star clusters in a sub-sample of galaxies. This will enable us to calibrate the star-formation rate in nearby galaxies on the basis of the compact radio source population, independent of the obscuration effects which can affect other measures of SFR. It should be possible to measure local star-formation rates around type Ia supernovae (undetected in the radio to date; (8)); where these thermonuclear supernovae have good light curves from e.g. the Palomar Transient Factory, it will be possible to calibrate the supernova peak luminosity SFR variation, very important cosmologically.

In addition, it has been known for some time that well-studied galaxies contain a population of radio transient sources of unknown origin (e.g. M82; (7)). It is possible that this survey will detect more of these events, helping to pin down their nature.

Accretion: the survey will provide a complete census of AGN activity and jet structure in local galaxies of all types, including starburst, LINER, and quiescent galaxies, as well as known AGN. LeMMINGS will probe several orders of magnitude lower in radio power than previous surveys, and will have sufficient resolution ( $\sim$ few pc) to separate star-formation and AGN/jet activity. This will help answer the question of whether AGN or supernovae produce more energy feedback into their environments.

Jet models predict a relationship between the X-ray and radio luminosities, and the mass of the accreting object. The observed relationship currently has a large scatter, largely due to the flux measurements from low-resolution radio observations being contaminated by non-AGN emission within the beam. The observations from LeMMINGs, at 50-150 mas resolution, will greatly improve the statistics.

Neutral gas: the wide bandwidths of the new system, and the flexibility of the WIDAR correlator, make it easy to obtain sensitive continuum and spectral line data simultaneously. LeMMINGs will make use of this capability for the deep tier observations, providing data on a variety of astrophysically important lines for a small number of galaxies. Providing information on $\mathrm{HI}$ and $\mathrm{OH}$ (main, satellite, and excited lines), as well as $\mathrm{H}_{2} \mathrm{CO}, \mathrm{HCN}$, etc, the cubes will provide a rich source of data on absorption, emission and maser activity within the galaxies in the first sub-arcsecond, simultaneous, multi-line survey of its kind.

\section{The sample}

The survey is being carried out using a two-tiered approach. The majority of the galaxies will be imaged via snapshot observations (the shallow sample), but a small sub-sample of nearby 

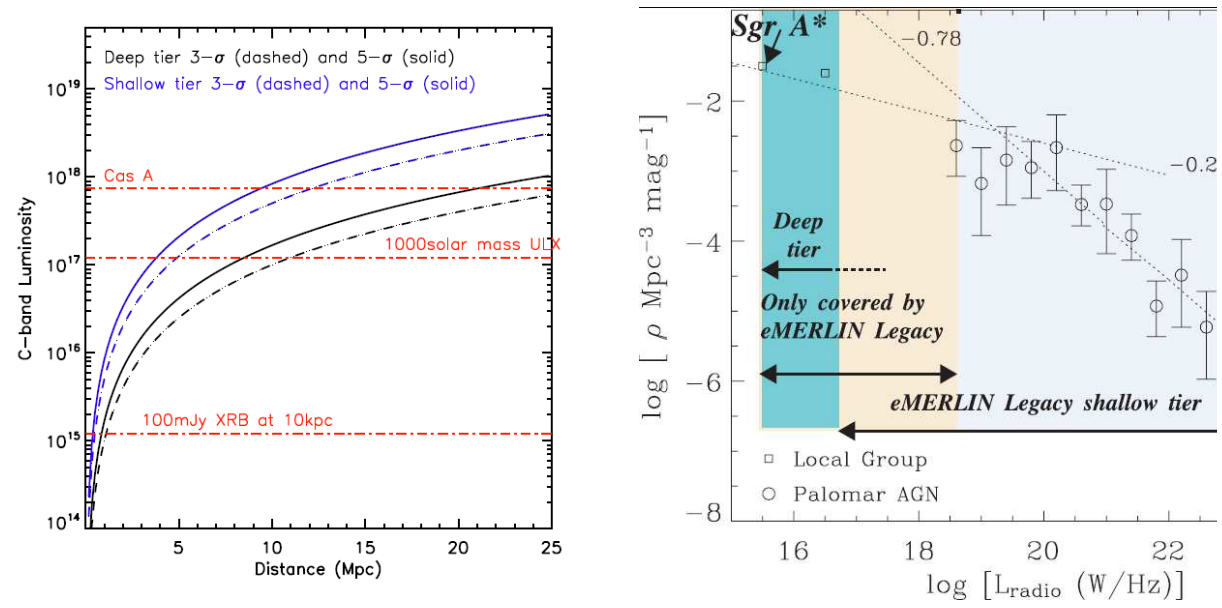

Figure 1: Left: C-band luminosity limits as a function of distance. Right: number density against radio power for AGN.

'interesting' galaxies with the best multi- $\lambda$ coverage will be imaged in longer observations (the deep sample) to reach a greater sensitivity.

The shallow sample is taken from the Palomar bright galaxy sample (6) selecting sources with a Declination $>20^{\circ}$ (290 galaxies). The sample is optically selected, so has no radio bias, has $\mathrm{B}_{\mathrm{T}}<$ $12.5 \mathrm{mag}$, and a median Distance of $20 \mathrm{Mpc}$. All of the galaxies in the sample have good multiwavelength coverage, with the sample having good overlap with existing major surveys such as SINGS, KINGFISH, THINGS, Galex etc. An additional aspect of the LeMMINGs project is a campaign to complete this multi- $\lambda$ coverage for galaxies in our sample where this ancillary data is missing or incomplete. LeMMINGs observations of the shallow sample consist of $\sim 48$ minutes per band, per target, with the observing time spread out over several hour angles to improve the u-v coverage.

The deep sample consists of a small subset of the shallow sample: a handful of nearby, wellstudied galaxies with a comprehensive amount of ancillary data already available. As such, they have considerable early science potential. Each galaxy in the deep sample will be observed with e-MERLIN for roughly five hours per band, per source. An additional technical goal of the early deep-tier observations (already made) is to test the image fidelity of snapshot observations with e-MERLIN in comparison with the longer observational runs of the full deep survey. The targets in the deep tier were selected to have a complex morphology and will be a good test of the snapshot imaging capability.

\section{The story so far}

Deep observations of some of the sample have already been carried out. The sources observed so far are: M82, a nearby prototypical starburst, IC10, a complex post-starburst dwarf irregular 


\begin{tabular}{cccccc} 
Tier & Band & Number of targets & $\begin{array}{c}\text { Sensitivity } \\
\mu \mathrm{Jy} / \mathrm{mb}\end{array}$ & $\begin{array}{c}\text { Luminosity } \\
\mathrm{W} / \mathrm{Hz}\end{array}$ & $\begin{array}{c}\text { Time on source } \\
\text { hours }\end{array}$ \\
\hline shallow & $\mathrm{L}$ & 290 & 38 & $1.8 \times 10^{18}$ & 0.8 \\
shallow & $\mathrm{C}$ & 290 & 15 & $7.2 \times 10^{17}$ & 0.8 \\
deep & $\mathrm{L}$ & 6 & 8 & $7.5 \times 10^{16}$ & 4.8 \\
deep & $\mathrm{C}$ & 6 & 3 & $2.8 \times 10^{16}$ & 4.8 \\
\hline
\end{tabular}

Table 1: The total LeMMINGs allocation is 810 hours of observing time, split over several semesters. This table shows how the time is divided between the two tiers, with the target sensitivity for each band and the resulting luminosity sensitivity (at the median distance of the sample).

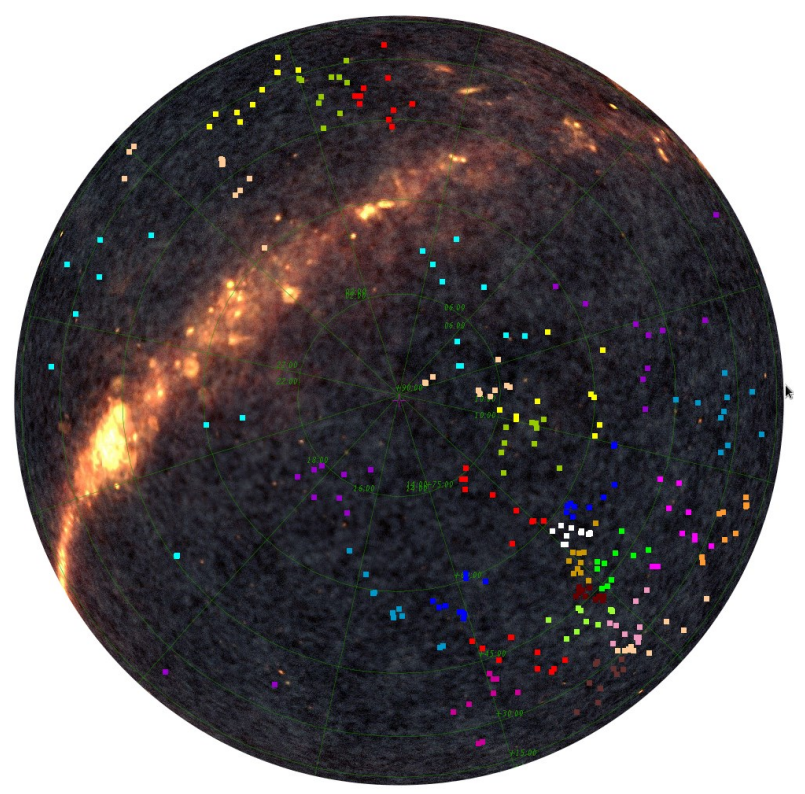

Figure 2: The sky positions of the LeMMINGs shallow sample, consisting of 280 galaxies. These target observations are being observed in blocks of ten sources. Individual scheduling blocks of sources are repressented by different coloured symbols.

galaxy, IC342, a nearby dwarf, and NGC2146, another local starburst.

Observations of all 280 galaxies in the shallow observations have been scheduled and are in the e-MERLIN queue. The sample has been split into blocks of 10 galaxies (plus calibrators) per observation, clustered on the sky to minimise overheads. To date, a handful of blocks have been observed at both bands, and the data reduction is being carried out by various members of the collaboration.

\section{4. $M 82$}

Observed extensively over the last two decades, M82 is known to contain over 50 compact radio sources $(1 ; 3 ; 4 ; 5)$. These include compact supernova remnants as well as more diffuse HII regions, and more unusual transient sources (7). Just after the type Ia supernova 2014J was 


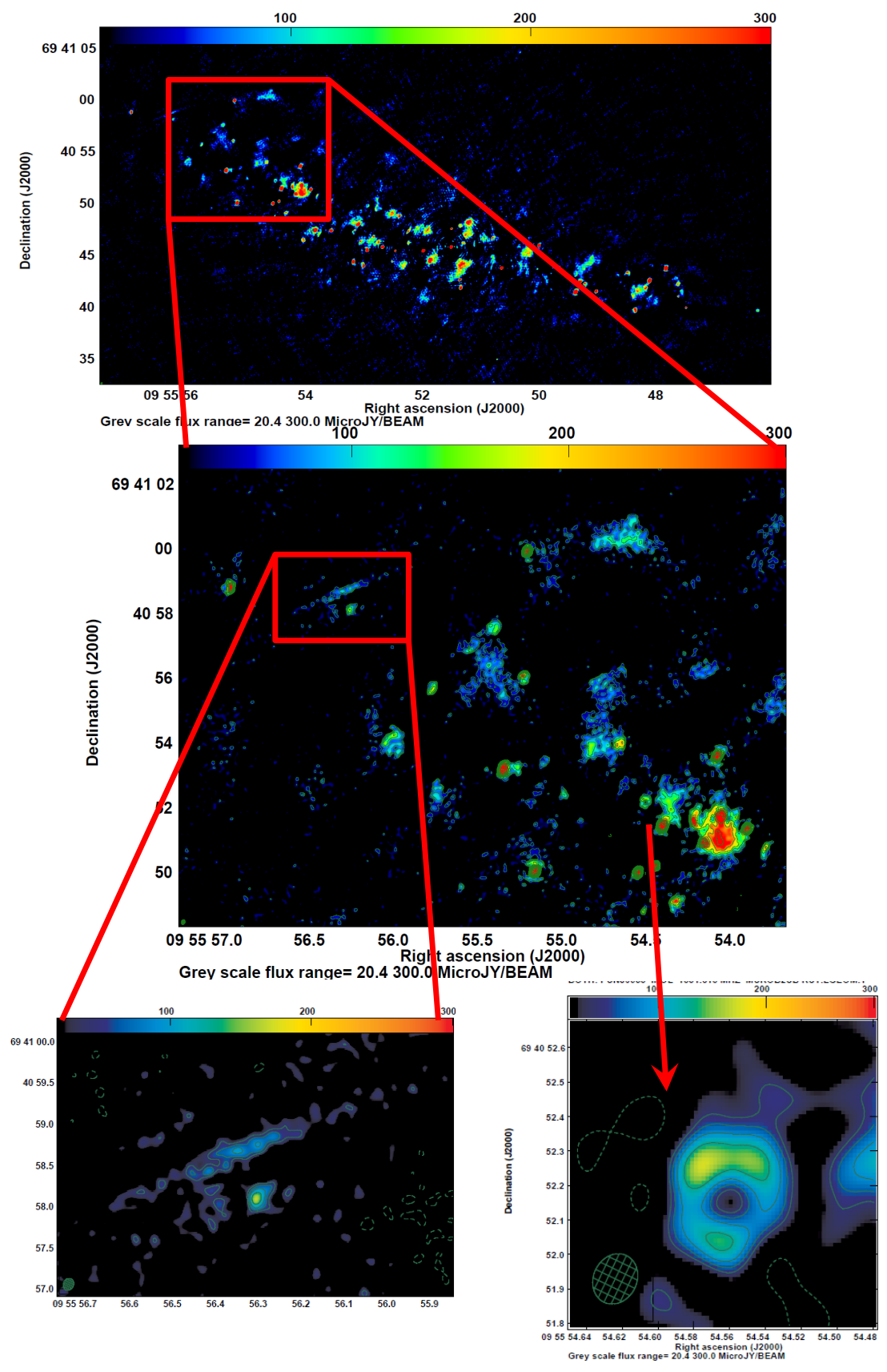

Figure 3: Deepest e-MERLIN images of the starburst galaxy M82 at L-band made to date. The top image shows the radio emission from multiple compact source across the entire central kpc of M82. Low images show 'zoom-in' to the north-eastern region of the galaxy, demonstrating the high resolution and high sensitivity of these e-MERLIN observations. Within these data numerous previously unreported sources, such as the faint RSN shell (bottom-right) and striking extended linear shock feature (bottom-left, see also (9)) have been detected. 
reported in M82, new deep observations were made with e-MERLIN to search for radio emission at both L- and C-band (8). In addition to putting sensitive limits on the radio flux from this event, these observations have provided extremely sensitive images ( $\mathrm{rms} 13.6$ and $12.4 \mu \mathrm{Jy} \mathrm{bm}^{-1}$ at $\mathrm{L}$ and C-band resectively) of the galaxy (Fig. 3). These high fidelity, data are now allowing a detailed analysis of the compact radio source population within this galaxy, and provide a taster of the quality and richness of the science data now being provided by e-MERLIN and the LeMMINGs legacy project.

\section{Summary and future progress}

The LeMMINGs e-MERLIN legacy project aims to provide a comprehensive high resolution radio survey of galaxies within the local Universe, which is a key driver for both the existing and next generations of observing facilities (2). In doing so it will address some of the fundamental astrophysical questions regarding levels of star-formation and accretion processes while also revealing their interactions on poorly explored spatial scales in a large, statistical sample of galaxies. In particular, this legacy programme will make a complete imaging census of both AGN activity and jet structures and radio sources relating to key star-formation phases across all galaxy types and luminosities in the local Universe.

\section{Acknowledgements}

e-MERLIN is the UK's National Radio Interferometric facility, operated by the University of Manchester on behalf of the Science and Technology Facilities Council (STFC). JW and DW acknowledges receipt of STFC studentships.

\section{References}

[1] Beswick, R. et al. 2006, MNRAS, 369, 1221

[2] Beswick, R. et al. "SKA studies of nearby galaxies: star-formation, accretion processes and molecular gas across all environments", in proceedings of "Advancing Astrophysics with the Square Kilometre Array", 2015, PoS (AASKA14) 70

[3] Fenech et al, 2008, MNRAS, 391, 1384

[4] Fenech et al, 2010, MNRAS, 408, 607

[5] Gendre et al. 2013, MNRAS, 431, 1107

[6] Ho et al. 1997, ApJ, 487, 568

[7] Muxlow et al 2010 MNRAS, 404, 109

[8] Perez Torres et al 2014, ApJ, 792, 38

[9] Varenius, E. et al 2015, A\&A, in press 